\title{
Association between perioperative fluid administration and postoperative outcomes: a 20-year systematic review and a meta-analysis of randomized goal-directed trials in major visceral/noncardiac surgery
}

\author{
Antonio Messina $a^{1,2^{*}} \mathbb{D}$, Chiara Robba $^{3,4}$, Lorenzo Calabrò ${ }^{1}$, Daniel Zambelli ${ }^{1}$, Francesca lannuzzi ${ }^{4}$, \\ Edoardo Molinari ${ }^{4}$, Silvia Scarano ${ }^{4}$, Denise Battaglini ${ }^{4}$, Marta Baggiani ${ }^{5}$, Giacomo De Mattei ${ }^{6}$, Laura Saderi ${ }^{7}$, \\ Giovanni Sotgiu ${ }^{7}$, Paolo Pelosi ${ }^{3,4}$ and Maurizio Cecconi ${ }^{1,2}$
}

\begin{abstract}
Background: Appropriate perioperative fluid management is of pivotal importance to reduce postoperative complications, which impact on early and long-term patient outcome. The so-called perioperative goal-directed therapy (GDT) approach aims at customizing perioperative fluid management on the individual patients' hemodynamic response. Whether or not the overall amount of perioperative volume infused in the context of GDT could influence postoperative surgical outcomes is unclear.
\end{abstract}

Methods: We conducted a systematic review and meta-analysis of randomized controlled trials (RCTs) comparing the efficacy of GDT approach between study population and control group in reducing postoperative complications and perioperative mortality, using MEDLINE, EMBASE and the Cochrane Controlled Clinical trials register. The enrolled studies were grouped considering the amount infused intraoperatively and during the first $24 \mathrm{~h}$ after the admission in the critical care unit (perioperative fluid).

Results: The metanalysis included 21 RCTs enrolling 2729 patients with a median amount of perioperative fluid infusion of $4500 \mathrm{ml}$. In the studies reporting an overall amount below or above this threshold, the differences in postoperative complications were not statically significant between controls and GDT subgroup [43.4\% vs. 34.2\%, $p$ value $=0.23$ and $54.8 \%$ vs. $39.8 \% ; ~ p$ value $=0.09$, respectively]

Overall, GDT reduced the overall rate of postoperative complications, as compared to controls [pooled risk difference $(95 \% \mathrm{Cl})=-0.10(-0.14,-0.07) ; \mathrm{Chi}_{2}=30.97 ; p$ value $\left.<0.0001\right]$, but not to a reduction of perioperative mortality [pooled risk difference $(95 \% \mathrm{Cl})=-0.016(-0.0334 ; 0.0014) ; p$ value $=0.07$ ]. Considering the rate of organ-related postoperative events, GDT did not reduce neither renal ( $p$ value $=0.52$ ) nor cardiovascular ( $p$ value $=0.86$ ) or pulmonary ( $p$ value $=0.14$ ) or neurological ( $p$ value $=0.44$ ) or infective $(p$ value $=0.12)$ complications.

Conclusions: Irrespectively to the amount of perioperative fluid administered, GDT strategy reduces postoperative complications, but not perioperative mortality.

\footnotetext{
*Correspondence: antonio.messina@humanitas.it

${ }^{1}$ Humanitas Clinical and Research Center - IRCCS, Rozzano, MI, Italy

Full list of author information is available at the end of the article
} permits use, sharing, adaptation, distribution and reproduction in any medium or format, as long as you give appropriate credit to the original author(s) and the source, provide a link to the Creative Commons licence, and indicate if changes were made. The images or other third party material in this article are included in the article's Creative Commons licence, unless indicated otherwise in a credit line to the material. If material is not included in the article's Creative Commons licence and your intended use is not permitted by statutory regulation or exceeds the permitted use, you will need to obtain permission directly from the copyright holder. To view a copy of this licence, visit http://creativecommons.org/licenses/by/4.0/. The Creative Commons Public Domain Dedication waiver (http://creativeco mmons.org/publicdomain/zero/1.0/) applies to the data made available in this article, unless otherwise stated in a credit line to the data. 
Trial Registration: CRD42020168866; Registration: February 2020

https://www.crd.york.ac.uk/prospero/display_record.php?RecordID $=168866$

Keywords: Fluids, Surgery, Systematic review, Metanalysis, Perioperative goal-directed therapy, Postoperative complications

\section{Introduction}

Postoperative complications occur in a significant proportion of patients undergoing surgery [1-3], leading to mortality of about $4 \%$ in Europe [4], and having a significant impact on long-term morbidity and, in turn, on health and financial systems [5, 6]. Several aspects including preoperative frailty, intraoperative management and events and postoperative care may influence the risk of developing postoperative complications.

In this context, the optimization of perioperative fluid management plays a key role in maintaining tissue fluid and electrolyte homeostasis and euvolemia, while avoiding inadequate tissue perfusion and fluid overload, which have been both associated with worse clinical outcomes in surgical patients [7-13]. The most effective perioperative fluid management is unclear [14-17]. The Enhanced Recovery After Surgery (ERAS) pathways to support early recovery among patients undergoing major surgery recommend a restrictive approach aiming for the perioperative "zero-balance" [14]. In contrast, recent findings suggest that this approach could be detrimental, suggesting a moderately positive fluid balance of 1 to $2 \mathrm{~L}$ at the end of surgery [16].

However, regardless of the definitions adopted, perioperative fluid balance may result from either preoperative fixed fluid targets (i.e., overall fluid balance below a predetermined cutoff), or, as part of a protocol-based fluid administration. The so-called perioperative goal-directed therapy (GDT) is based on the purpose of balancing the increased oxygen demand during surgery, by the use of flow-based hemodynamic parameters, to achieve specific hemodynamic endpoints rather than a predetermined perioperative fluid balance $[8,18]$. Adopting a GDT approach, perioperative fluid balance is the effect of the individual response to fluid administration, being titrated on the hemodynamic response to each fluid bolus. However, the evidence regarding the effectiveness of perioperative GDT approaches is still inconclusive [8, 19-21].

We conducted a systematic review and meta-analysis of RCTs to assess whether the amount of perioperative volume administered by means of a GDT approach (defined as the quantity infused in intraoperative time and the first $24 \mathrm{~h}$ after the admission to the critical care unit) could influence postoperative outcomes. To address this point, we stratified the included studies considering the median amount of perioperative fluid given to patients receiving GDT, as compared to the controls. Secondarily, we assessed the overall effect of GDT on postoperative overall and organ-specific complications (i.e. renal, cardiovascular, pulmonary, neurological and infective), and perioperative mortality.

\section{Material and method}

We adhered to the Preferred Reporting Items for Systematic Reviews and Meta-Analysis-Protocols (PRISMA-P) guidelines [22] (Additional file 1: Table S1). The study protocol was registered with the International Prospective Register of Systematic Reviews (PROSPERO) in June 2020 (CRD42020168866).

\section{Data sources and search strategy}

A systematic literature search was performed including the following databases: PUBMED ${ }^{\circledR}$, EMBASE $^{\circledR}$ and the Cochrane Controlled Clinical trials register. The search was performed using the terms: ('goal-directed therapy' OR ('goal directed' AND ('therapy'/exp OR therapy)) OR 'goal-oriented therapy' OR ('goal oriented' AND ('therapy'/exp OR therapy)) OR 'goal-targeted therapy' OR ('goal targeted' AND ('therapy'/exp OR therapy))) AND ('surgery'/exp OR surgery) with filters for randomized trials.

Articles written in English and published from January 1, 2000, to December 31, 2019, in indexed scientific journals were considered. Editorials, commentaries, letters to editor, opinion articles, reviews, meeting abstracts were excluded. Only RCTs recruiting adult surgical populations using GDT approaches, reporting on morbidity (rate of postoperative complications) and/or mortality as primary or secondary outcomes were included. Studies focused on cardiac, trauma/orthopedic, pediatric, obstetric or neurosurgeries were excluded. References of selected papers, review articles, commentaries and editorials on this topic were also reviewed to identify other studies of interest missed during the primary search. When multiple publications of the same research group/ center described potentially overlapping cohorts, the more recent publications were selected.

GDT strategy was defined as a modality of perioperative treatment including the use of both (1) hemodynamic monitoring and (2) therapies (fluids and/or inotropes and vasopressors, alone or together) aimed at manipulating hemodynamic parameters during the 
perioperative period to achieve a predetermined hemodynamic endpoint(s). The step-by-step perioperative protocol based on patient-specific hemodynamic data retrieved from both a hemodynamic monitor (irrespective to the invasiveness) or surrogates of peripheral delivery/extraction of oxygen (i.e. lactate, central venous oxygen saturation, capillary refill time) had to be detailed in the selected studies.

The GDT goals might include both the optimization of either systemic flow or pressure parameters (i.e., mean systemic pressure or stroke volume), or hemodynamic indexes (i.e., stroke or pulse pressure variations). Only RCTs comparing GDT strategy versus a single control population were selected. We considered perioperative fluid administration as the overall amount of fluids infused in a period including intraoperative time and the first $24 \mathrm{~h}$ after admission to a critical care unit [15].

\section{Data abstraction and quality assessment}

Three couples of examiners independently evaluated titles and abstracts. The articles were then subdivided into three subgroups: "included" and "excluded" (if the two examiners agreed with the selection) or "uncertain" (in case of disagreement). In the case of "uncertain" classification, discrepancies were resolved by further examination performed by two expert authors (A.M. and C.R.). We used a standardized electronic spreadsheet (Microsoft Excel, V 14.4.1; Microsoft, Redmond, WA) to extract data from all included studies, recording: trial characteristics (i.e. number of centers, country), patient population (i.e. demographics, type of surgery, baseline illness severity scores), intraoperative monitoring and interventions (i.e. mechanical ventilation characteristics, monitoring technology used, goal-directed therapy targets, type and amount of fluid) and clinical outcomes (i.e. mortality, morbidity related to organ-specific function or infections) (Additional file 1: Table S2). When necessary, the corresponding authors of the included studies were contacted to obtain missing data related to trial demographics, methods and outcomes.

\section{Risk of bias assessment in the included studies.}

The internal validity of the included studies was assessed by two expert authors (A.M. and C.R.), and discrepancies were resolved by a third author (M.C.) by using the RoB 2: a revised Cochrane Collaboration's risk-of-bias tool for randomized trials [23]. The RoB 2 considers five bias domains: (1) the randomization process; (2) the deviations from intended interventions; (3) missing outcome data; (4) measurement of the outcome; (5) selection of the reported results. Finally, overall risk of bias was calculated and, accordingly, studies were classified as highrisk/some concerns/low-risk.

\section{Statistical analysis}

Descriptive analysis was carried out: The statistical unit of observation for all the selected variables was the single study and not the patient. Means with standard deviations (SD) described for continuous variables.

The meta-analysis included only those studies reporting the rate of overall perioperative complications, according to the definition adopted in each study (i.e., overall, severe, not-severe). For the primary outcome, the association between perioperative fluid administration and complications was assessed considering the median fluid dose given to the subgroups of patients receiving GDT, as compared to controls, and predefined cutoffs of postoperative complications frequencies (i.e., $<30 \%$; $30-50 \%$; $>50 \%)$. For the secondary outcomes, we considered the effect of GDT approach on (1) postoperative overall rate of complications (number of patients having at least one complication); (2) organ-related events [renal, cardiac, pulmonary non-infective (i.e., pulmonary embolism), neurological and infective (including either sepsis/septic shock or organ-specific infections)]; (3) perioperative mortality.

Publication bias was graphically evaluated using funnel plots. Heterogeneity was measured using $Q$ and $I^{2}$ tests, which were considered significant when the $p$ value was $<0.1$ and $I^{2}>50 \%$. Random or fixed effect models were used based on the expected heterogeneity. According to Higgins et al. [24], $I^{2}$ values around $25 \%, 50 \%$ and $75 \%$ represented low, moderate and high heterogeneity.

The statistical software STATA ${ }^{\circledR}$ version 16 (StataCorp, College Station, TX, USA) and StatsDirect version 3.2.7 were used to perform all the statistical computations.

\section{Results}

The electronic search identified 1,834 titles after removing duplicate studies. Experts evaluated and solved the inclusion of $25(1.3 \%)$ potentially relevant studies because of disagreement between the examiners. The meta-analysis was performed on 21 RCTs enrolling 4753 and analyzing 2729 patients (Fig. 1 and Table 1). Excluded studies are reported in Additional file 1: Table S3. The bias risk assessment reported "low risk" for $5(22.7 \%)$ and "some concerns" for 16 (76.2\%) of the included studies, mostly related to the selection of the reported results (Additional file 1: Figure S1).

\section{General characteristics of the GDT subgroups and perioperative fluid administration.}

Patient characteristics were consistent among the studies. Patients receiving GDT had a mean (SD) age of 62 (5) years (males $=59 \%$ ), a mean (SD) body mass index of $26 \pm 2 \mathrm{~kg} / \mathrm{m}^{2}$, and had a mean (SD) surgical time of 238 


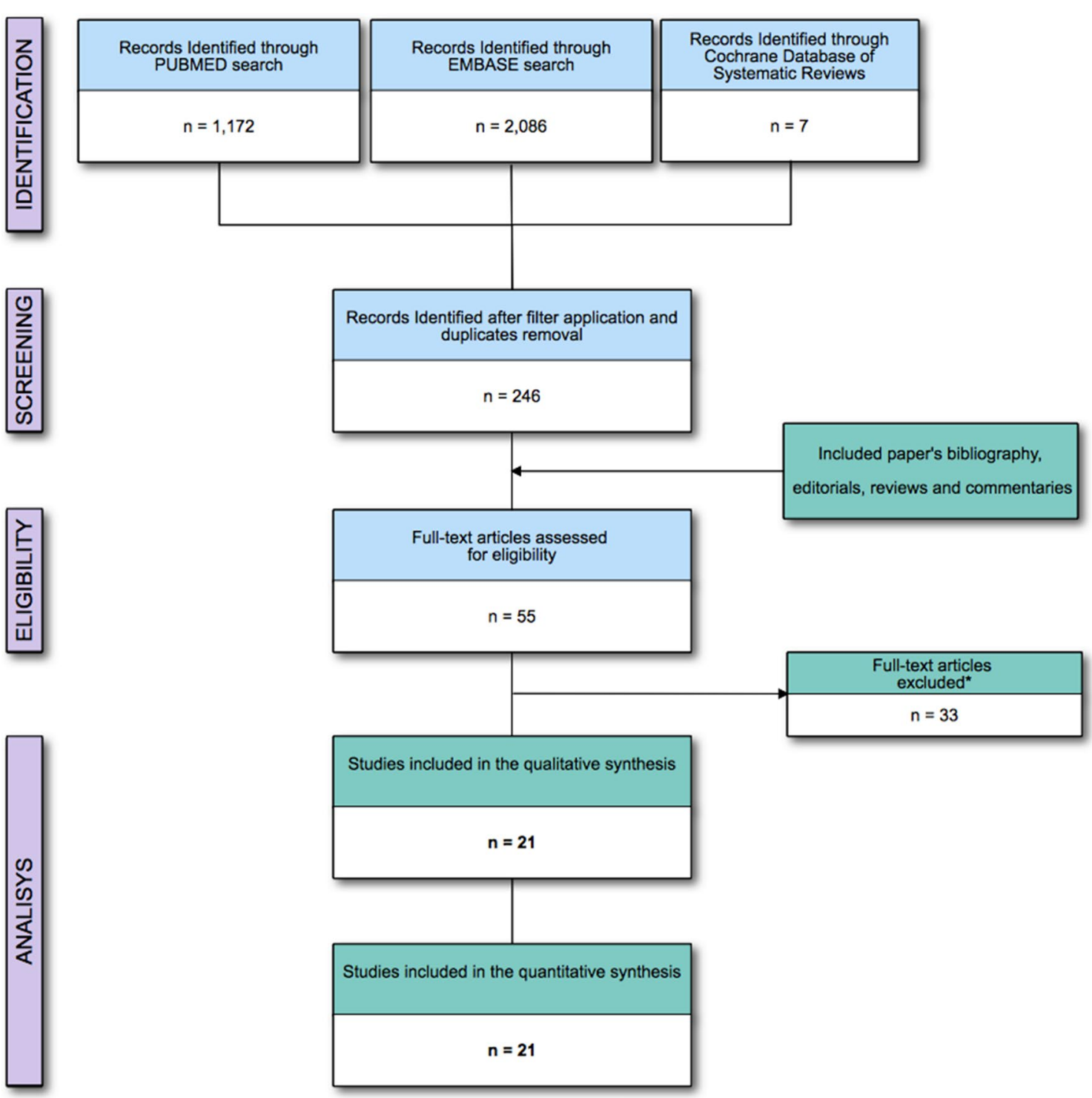

Fig. 1 Flow of the studies. * = Not fitting eligibility criteria full-text articles excluded a reported in the Additional file 1: Table S3

(91) minutes. In the controls subgroup, mean (SD) age was 66 (6) years (males $=56.4 \%$ ) with mean $(\mathrm{SD})$ body mass index of $26 \pm 2$ and had a mean (SD) surgical time of 236 (81) minutes (see also Tables 1 and 2).

The summary of the intraoperative amount of fluids infused is reported in Table 2. Intraoperatively, the GDT subgroup received a mean (SD) of 1632 (928) $\mathrm{ml}$ of crystalloids and of 1053 (603) $\mathrm{ml}$ of colloids (51\% of the overall amount). In contrast, controls received a mean (SD) of 1977 (1142) ml of crystalloids, and of 758 (586) ml of colloids (44\% of the overall amount). Nine studies [25-33] reported a mean (SD) intraoperative blood transfusion of $320 \mathrm{ml}$ (297) and $340 \mathrm{ml}$ (299) in GDT and controls, respectively. Only three studies [27, 29, 34] reported the cumulative fluid balance of GDT and control groups during the first two postoperative days (Additional file 1: Table S4).

\section{Effect of fluid administration on the rate of complications}

The median amount of perioperative fluid infusion in the included studies was $4500 \mathrm{ml}$. Differences in the incidence $(95 \% \mathrm{CI})$ of postoperative complications between patients receiving GDT and controls in those studies reporting a volume below or above this thresholds were not significant $[43.4 \%(34.2 ; 54.9)$ vs. $34.2 \%(22.5 ; 45.8), p$ 


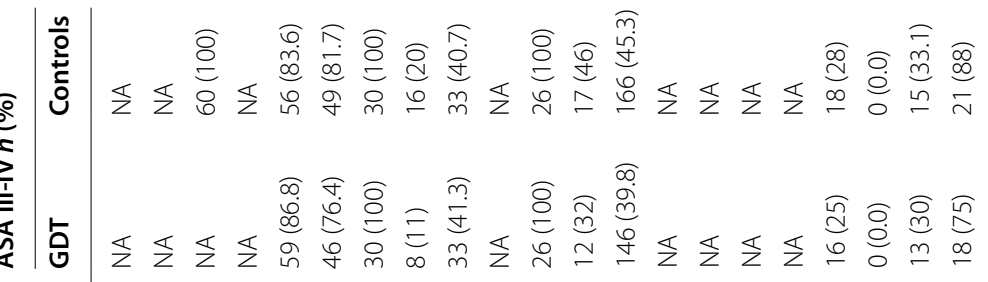

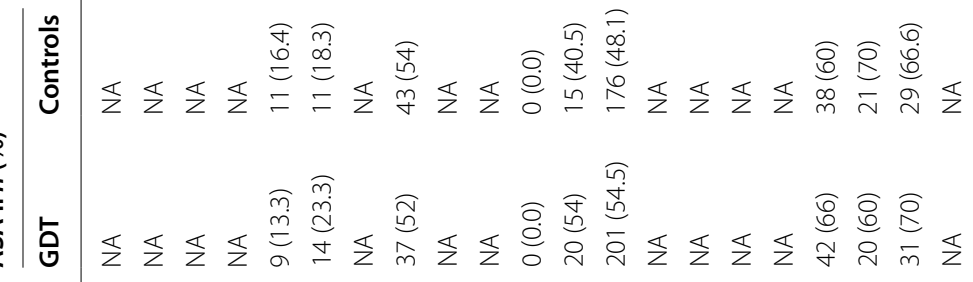

U

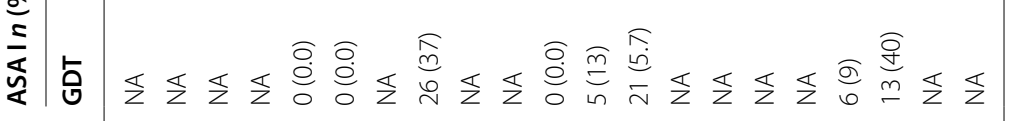

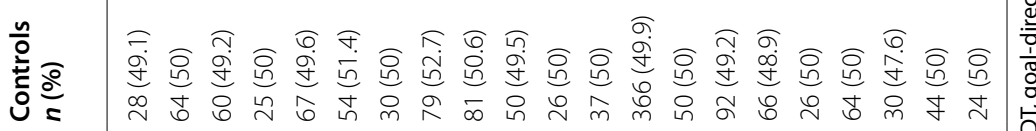

等

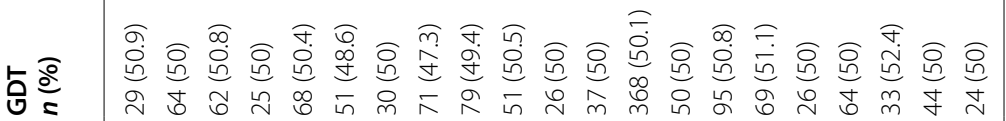

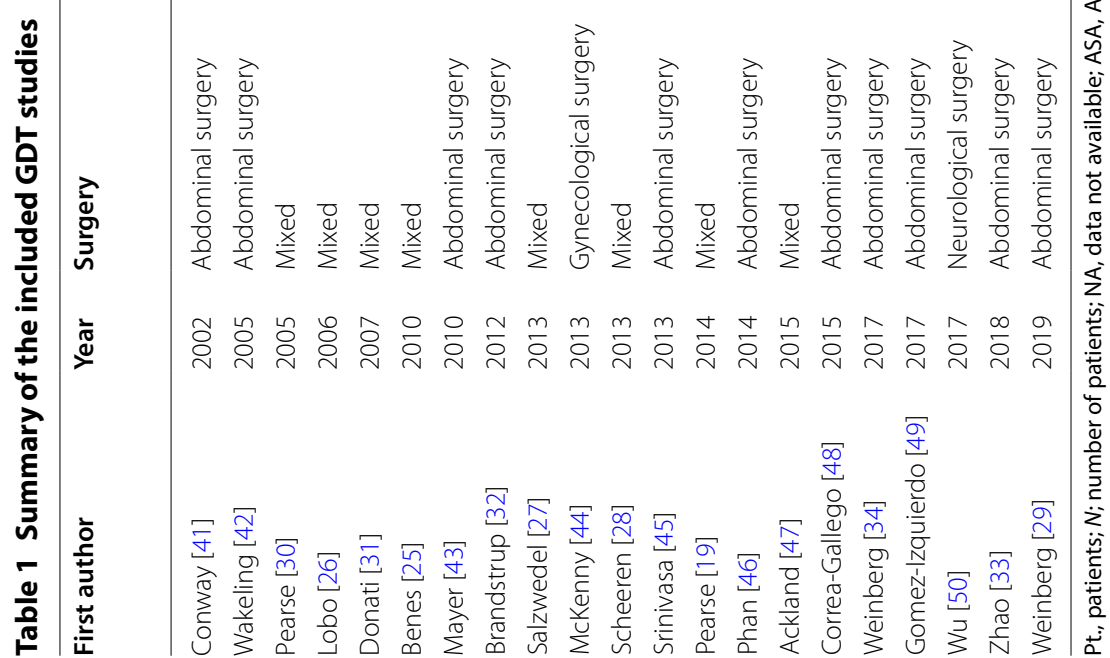


Table 2 Characteristics of GDT/control subgroups hemodynamic protocol

\begin{tabular}{|c|c|c|c|c|c|c|c|}
\hline & Group & $\begin{array}{l}\text { Bolus } \\
\text { (amount) }\end{array}$ & $\begin{array}{l}\text { Bolus } \\
\text { (type) }\end{array}$ & $\begin{array}{l}\text { Vasopressor } \\
\text { (type, \% of pt.) }\end{array}$ & Hemodynamic goal & $\begin{array}{l}\text { Transfusion } \\
\text { (\% of pt.) }\end{array}$ & $\begin{array}{l}\text { Hemodynamic } \\
\text { monitor }\end{array}$ \\
\hline \multirow[t]{2}{*}{ Conway [41] } & GDT & $3 \mathrm{ml} / \mathrm{kg}$ & Voluven $^{\circledR}$ & NA & $\begin{array}{l}\text { SV }>10 \% \text { after FC } \\
\text { FTC }<0.35 \text { s }\end{array}$ & NA & $\mathrm{ODP}\left(\mathrm{TECO}^{\circledR}\right)$ \\
\hline & Control & NA & NA & NA & NA & NA & $\begin{array}{l}\text { Standard } \\
\text { monitoring }\end{array}$ \\
\hline \multirow[t]{2}{*}{ Wakeling [42] } & GDT & $250 \mathrm{ml}$ & $\begin{array}{l}\text { Haemaccel }{ }^{\circledR} \\
\text { Gelofusine }{ }^{\circledR}\end{array}$ & NA & $\begin{array}{l}\text { Repeated FC until } \\
\text { SV response }>10 \% \text { or } \\
\quad \text { CVP }>3 \mathrm{mmHg}\end{array}$ & NA & ODP_CardioQ \\
\hline & Control & Standard care & Standard care & NA & $\begin{array}{l}\text { CVP 12- } 15 \mathrm{mmHg} \\
\text { Oxygen Delivery } \\
\text { Index >600 ml/ } \\
\mathrm{min} / \mathrm{m}^{2}\end{array}$ & NA & ODP_CardioQ \\
\hline \multirow[t]{2}{*}{ Pearse [30] } & GDT & $250 \mathrm{ml}$ & Gelofusine ${ }^{\circledR}$ & Dopexamine (all) & $\begin{array}{l}\text { SV response }>10 \% \text { for } \\
20 \mathrm{~min} \\
\text { CVP } \\
\text { increase }>2 \mathrm{mmHg} \\
\text { for } 20 \mathrm{~min}\end{array}$ & NA & LiDCOplus \\
\hline & Control & $250 \mathrm{ml}$ & Gelofusine ${ }^{\circledR}$ & NA & $\begin{array}{l}\text { CVP increase of at } \\
\text { least } 2 \mathrm{mmHg} \\
\text { for } 20 \mathrm{~min}\end{array}$ & NA & $\begin{array}{l}\text { Standard } \\
\text { monitoring }\end{array}$ \\
\hline \multirow[t]{2}{*}{ Lobo [26] } & GDT & $\begin{array}{l}1000 \mathrm{ml} \\
500 \mathrm{ml}\end{array}$ & $\begin{array}{l}\text { Crystalloids } \\
\text { Gelatine }\end{array}$ & Dobutamina; 8\% & $\begin{array}{l}\text { Oxygen Delivery } \\
\text { Index }<600 \mathrm{ml} / \\
\mathrm{min} / \mathrm{m}^{2} \\
\text { PAOP }<16 \mathrm{mmHg}\end{array}$ & $44 \%$ & PAC \\
\hline & Control & $\begin{array}{l}1000 \mathrm{ml} \\
500 \mathrm{ml}\end{array}$ & $\begin{array}{l}\text { Crystalloids } \\
\text { Gelatine }\end{array}$ & Dobutamina; 100\% & $\begin{array}{l}\text { Oxygen Delivery } \\
\text { Index }<600 \mathrm{ml} / \\
\mathrm{min} / \mathrm{m}^{2} \\
\text { PAOP }<16 \mathrm{mmHg}\end{array}$ & $48 \%$ & PAC \\
\hline \multirow[t]{2}{*}{ Donati [31] } & GDT & NA & $\begin{array}{l}\text { Colloids UN } \\
(250-1000 \mathrm{ml})\end{array}$ & $\begin{array}{l}\text { Dobutamine; } \\
\quad 44.1 \%\end{array}$ & $\begin{array}{l}\mathrm{O}_{2} \mathrm{ER}<27 \% \\
M A P>80 \mathrm{~mm} \mathrm{Hg} \\
U O>0.5 \mathrm{ml} / \mathrm{kg} / \mathrm{h} \\
\text { CVP } 8-12 \mathrm{~cm} \mathrm{H}_{2} \mathrm{O}\end{array}$ & NA & $\mathrm{O}_{2} \mathrm{ER}$ \\
\hline & Control & NA & $\begin{array}{l}\text { Colloids UN } \\
(250-1000 \mathrm{ml})\end{array}$ & Dobutamine; $4.5 \%$ & $\begin{array}{l}M A P>80 \mathrm{~mm} \mathrm{Hg} \\
U O>0.5 \mathrm{ml} / \mathrm{kg} / \mathrm{h} \\
\text { CVP } 8-12 \mathrm{~cm} \mathrm{H}_{2} \mathrm{O}\end{array}$ & NA & $\begin{array}{l}\text { Standard } \\
\text { monitoring }\end{array}$ \\
\hline \multirow[t]{2}{*}{ Benes [25] } & GDT & $3 \mathrm{ml} / \mathrm{kg}$ & $\begin{array}{l}\text { Voluven }{ }^{\circledR} \\
\text { Tetraspan }{ }^{\circledR}\end{array}$ & $\begin{array}{l}\text { Norepinephrine; } \\
\quad 13.7 \% \\
\text { Dobutamine; } 1.9 \%\end{array}$ & $\begin{array}{l}\text { SWV }<10 \% \\
\text { Cl/CVP changes } \\
\text { after FC }\end{array}$ & NA & Vigileo/FT \\
\hline & Control & NA & $\begin{array}{l}\text { Colloids UN } \\
\text { Crystalloids UN }\end{array}$ & $\begin{array}{l}\text { Norepinephrine, } \\
11.1 \% \\
\text { Dobutamine, 0\% }\end{array}$ & $\begin{array}{l}\mathrm{MAP}>65 \mathrm{~mm} \mathrm{Hg} \\
\mathrm{UO}>0.5 \mathrm{ml} / \mathrm{kg} / \mathrm{h} \\
\text { CVP } 8-115 \mathrm{cmH}_{2} \mathrm{O}\end{array}$ & NA & $\begin{array}{l}\text { Standard } \\
\text { monitoring }\end{array}$ \\
\hline \multirow[t]{2}{*}{ Mayer [43] } & GDT & $\begin{array}{l}500 \mathrm{ml} \text { (first) } \\
250 \mathrm{ml} \text { (further) }\end{array}$ & $\begin{array}{l}\text { Cristalloyds UN } \\
\text { Colloids UN }\end{array}$ & $\begin{array}{l}\text { Norepinephrine } \\
\text { (\%NA) } \\
\text { Dobutamine (\%NA) }\end{array}$ & $\begin{array}{l}\mathrm{Cl}<2.5 \mathrm{~L} / \mathrm{min} / \mathrm{m}^{2} \\
\mathrm{MAP}>65 \mathrm{~mm} \mathrm{Hg} \\
\mathrm{SVI}>/<35 \mathrm{ml} / \mathrm{min} / \\
\mathrm{m}^{2}\end{array}$ & NA & Vigileo/FT \\
\hline & Control & $\begin{array}{l}500 \mathrm{ml} \text { (first) } \\
250 \mathrm{ml} \text { (further) }\end{array}$ & $\begin{array}{l}\text { Cristalloyds UN } \\
\text { Colloids UN }\end{array}$ & NA & $\begin{array}{l}\text { MAP } 65-90 \mathrm{~mm} \mathrm{Hg} \\
\mathrm{UO}>0.5 \mathrm{ml} / \mathrm{kg} / \mathrm{h} \\
\text { CVP } 8-12 \mathrm{~cm} \mathrm{H}_{2} \mathrm{O}\end{array}$ & NA & $\begin{array}{l}\text { Standard } \\
\text { monitoring }\end{array}$ \\
\hline \multirow[t]{2}{*}{ Brandstrup [32] } & GDT & $200 \mathrm{ml}$ & Voluven ${ }^{\circledR}$ & $\begin{array}{l}\text { Different vasopres- } \\
\text { sors (\%NA) }\end{array}$ & $\begin{array}{l}\text { Repeated FC until } \\
\text { SV response }>10 \%\end{array}$ & NA & ODP_-CardioQ \\
\hline & Control & $200 \mathrm{ml}$ & Voluven ${ }^{\circledR}$ & $\begin{array}{l}\text { Different vasopres- } \\
\text { sors (\%NA) }\end{array}$ & $\begin{array}{l}\text { MAP > } 60 \mathrm{mmHg} \\
\text { Hematocrit } 25 \text { and } \\
\quad 35 \%\end{array}$ & NA & ODP-CardioQ \\
\hline
\end{tabular}


Table 2 (continued)

\begin{tabular}{|c|c|c|c|c|c|c|c|}
\hline & Group & $\begin{array}{l}\text { Bolus } \\
\text { (amount) }\end{array}$ & $\begin{array}{l}\text { Bolus } \\
\text { (type) }\end{array}$ & $\begin{array}{l}\text { Vasopressor } \\
\text { (type, \% of pt.) }\end{array}$ & Hemodynamic goal & $\begin{array}{l}\text { Transfusion } \\
\text { (\% of pt.) }\end{array}$ & $\begin{array}{l}\text { Hemodynamic } \\
\text { monitor }\end{array}$ \\
\hline \multirow[t]{2}{*}{ Salzwedel [27] } & GDT & NA & NA & $\begin{array}{l}\text { Norepinephrine, } \\
32.9 \% \\
\text { Phenylephrine,0\% } \\
\text { Ephedrine, } 13.9 \% \\
\text { Dobutamine, } 41.8 \%\end{array}$ & $\begin{array}{l}\mathrm{PPV}<10 \% \\
\mathrm{Cl}>2.5 \mathrm{~L} / \mathrm{min} / \mathrm{m}^{2} \\
\mathrm{MAP}>65 \mathrm{mmHg}\end{array}$ & NA & ProAQT \\
\hline & Control & NA & NA & $\begin{array}{l}\text { Norepinephrine, } \\
3.5 \% \\
\text { Phenylephrine, } \\
4.9 \% \\
\text { Ephedrine, 9.8\% } \\
\text { Dobutamine, 0\% }\end{array}$ & $A D$ & NA & $\begin{array}{l}\text { Standard } \\
\text { monitoring }\end{array}$ \\
\hline \multirow[t]{2}{*}{ McKenny [44] } & GDT & $3 \mathrm{ml} / \mathrm{kg}$ & Voluven ${ }^{\circledR}$ & NA & $\begin{array}{l}\text { Repeated FC until } \\
\text { SV response }>10 \%\end{array}$ & $8 ; 16 \%$ & ODP \\
\hline & Control & Standard care & $A D$ & NA & $A D$ & $8 ; 16 \%$ & ODP \\
\hline \multirow[t]{2}{*}{ Scheeren [28] } & GDT & $200 \mathrm{ml}$ & Voluven ${ }^{\circledR}$ & $\begin{array}{l}\text { Norepinephrine } \\
(\% \mathrm{NA})\end{array}$ & $\begin{array}{l}\text { Repeated FC if } \\
\text { SWV }>10 \% \\
\text { Repeated FC until } \\
\text { SV response }>10 \%\end{array}$ & NA & Vigileo/FT \\
\hline & Control & Standard care & $\begin{array}{l}\text { Colloids UN } \\
\text { Crystalloids UN }\end{array}$ & $\begin{array}{l}\text { Norepinephrine } \\
(\% \mathrm{NA})\end{array}$ & Standard care & NA & Vigileo/FT \\
\hline \multirow[t]{2}{*}{ Srinivasa [45] } & GDT & $\begin{array}{l}7 \mathrm{ml} / \mathrm{kg} \text { FC first } \\
3 \mathrm{ml} / \mathrm{kg} \text { other }\end{array}$ & Gelofusine $^{\circledR}$ & 83.7\% (type UN) & $\begin{array}{l}\text { FTC } 0.35-0.4 s \\
\text { SV after FC }>10 \%\end{array}$ & $13,35 \%$ & ODP_CardioQ \\
\hline & Control & $\begin{array}{l}\text { Up to } 1500 \mathrm{ml} \text { Plasm } \\
\text { Up to } 1500 \mathrm{ml} \text { Gelof }\end{array}$ & $\begin{array}{l}\text { alyte }{ }^{\circledR} \\
\text { usine }^{\circledR}\end{array}$ & 91.8\% (type UN) & Standard care & $12,32 \%$ & ODP_CardioQ \\
\hline \multirow[t]{2}{*}{ Pearse [19] } & GDT & $250 \mathrm{ml}$ & Colloids UN & $\begin{array}{l}\text { Bolus } 82.2 \% \\
\text { Infusion } 28.1 \%\end{array}$ & $\begin{array}{l}\text { MAP } 60-100 \text { mmHg } \\
\text { SV optimization }\end{array}$ & NA & LiDCOrapid \\
\hline & Control & Standard care & Standard care & $\begin{array}{l}\text { Bolus } 74.8 \% \\
\text { Infusion 30\% }\end{array}$ & Standard care & NA & NA \\
\hline \multirow[t]{2}{*}{ Phan [46] } & GDT & $250 \mathrm{ml}$ & $\begin{array}{l}\text { Voluven }^{\circledR} \\
\text { Gelofusine }{ }^{\circledR} \\
\text { 4\% human albumin }\end{array}$ & NA & $\begin{array}{l}\text { Hypotension } \\
\mathrm{SVI}<35 \mathrm{ml} / \mathrm{m}^{2} \\
\mathrm{FTC}<0.3 \mathrm{~s}\end{array}$ & None & ODP \\
\hline & Control & $\begin{array}{l}\text { Blood loss replace- } \\
\text { ment } \\
\text { Hypotension not } \\
\text { responsive to } \\
\text { vasopressor }\end{array}$ & $\begin{array}{l}\text { Ringer lactate } \\
\text { UN Colloids }\end{array}$ & NA & none & None & $\begin{array}{l}\text { Standard } \\
\text { monitoring }\end{array}$ \\
\hline \multirow[t]{2}{*}{ Ackland [47] } & GDT & NA & Gelatine & $\begin{array}{l}\text { Vasopressor } 18 \\
\quad(19 \%)\end{array}$ & $\begin{array}{l}\text { Oxygen Delivery } \\
\text { Index pre-postop- } \\
\text { erative } \\
\text { Repeated FC until SV } \\
\text { response }>10 \%\end{array}$ & $25(26 \%)$ & LiDCOplus \\
\hline & Control & NA & Gelatine & $\begin{array}{l}\text { Vasopressor } 21 \\
\quad(23 \%)\end{array}$ & $\begin{array}{l}\mathrm{SCVO}_{2}>65 \% \\
\mathrm{MAP}>60 \mathrm{~mm} \mathrm{Hg} \\
\mathrm{UO}>0.5 \mathrm{~mL} / \mathrm{kg} / \mathrm{h} \\
\text { Oxygen Delivery } \\
\text { Index }\end{array}$ & $16(17 \%)$ & LiDCOplus \\
\hline \multirow[t]{2}{*}{ Correa-Gallego C } & GDT & $\begin{array}{l}\text { 1:1 blood loss replac } \\
\text { Albumin infusion }\end{array}$ & ement & (type NA) 58\% & $\begin{array}{l}\text { SW to a value } \leq 2 S D \\
\text { from baseline }\end{array}$ & $6 \%$ & EV1000 \\
\hline & Control & $\begin{array}{l}\text { 1:1 blood loss replac } \\
6 \mathrm{ml} / \mathrm{kg} / \mathrm{hr} \text { Crystallo }\end{array}$ & $\begin{array}{l}\text { ement } \\
\text { ds }\end{array}$ & (type NA) 58\% & $\begin{array}{l}\text { Hypotension systolic } \\
\mathrm{BP}<90 \mathrm{mmHg} \\
\text { Urine out- } \\
\text { put }<25 \mathrm{ml} / \mathrm{h}\end{array}$ & $2 \%$ & EV1000 \\
\hline \multirow[t]{2}{*}{ Weinberg } & GDT & $250 \mathrm{~mL}$ & $\begin{array}{l}\text { Hartmann or Plas- } \\
\text { maLyte } \\
4 \% \text { or } 20 \% \text { albumin }\end{array}$ & NA & $\begin{array}{l}\mathrm{SWV}<20 \% \\
\mathrm{MAP}<20 \% \text { of } \\
\text { baseline } \\
\mathrm{Cl}>2 \mathrm{~L} / \mathrm{kg} / \mathrm{m}^{2}\end{array}$ & NA & EV1000 \\
\hline & Control & $A D$ & $\begin{array}{l}\text { Hartmann or Plas- } \\
\text { maLyte } \\
4 \% \text { or } 20 \% \text { albumin }\end{array}$ & NA & $A D$ & NA & EV1000 \\
\hline
\end{tabular}


Table 2 (continued)

\begin{tabular}{|c|c|c|c|c|c|c|c|}
\hline & Group & $\begin{array}{l}\text { Bolus } \\
\text { (amount) }\end{array}$ & $\begin{array}{l}\text { Bolus } \\
\text { (type) }\end{array}$ & $\begin{array}{l}\text { Vasopressor } \\
\text { (type, \% of pt.) }\end{array}$ & Hemodynamic goal & $\begin{array}{l}\text { Transfusion } \\
\text { (\% of pt.) }\end{array}$ & $\begin{array}{l}\text { Hemodynamic } \\
\text { monitor }\end{array}$ \\
\hline \multirow{2}{*}{$\begin{array}{l}\text { Gomez-Izquierdo } \\
\text { [49] }\end{array}$} & GDT & $200 \mathrm{~mL}$ & Voluven ${ }^{\circledR}$ & NA & SV optimization & NA & ODP \\
\hline & Control & $A D$ & $\begin{array}{l}\text { Ringer Lactate } \\
\text { Voluven }\end{array}$ & NA & $A D$ & NA & ODP \\
\hline \multirow[t]{2}{*}{ Wu [50] } & GDT & $50 \mathrm{ml}$ & Colloids (UN) & $\begin{array}{l}\text { Ephedrine, phe- } \\
\text { nylephrine; } \\
\text { dobutamine }\end{array}$ & $S W=12 \%$ & NA & Vigileo/FT \\
\hline & Control & $50 \mathrm{ml}$ & Colloids (UN) & $\begin{array}{l}\text { Ephedrine, phe- } \\
\text { nylephrine }\end{array}$ & $\begin{array}{l}\mathrm{CVP}>8 \mathrm{mmHg} \\
\mathrm{MAP}<80 \% \text { of base- } \\
\text { line value }\end{array}$ & NA & Vigileo/FT \\
\hline \multirow[t]{2}{*}{ Zhao [33] } & GDT & $200 \mathrm{~mL}$ & Different Colloids & MAP/Cl guided & $\begin{array}{l}S W V<15 \% \\
M A P \geq 65 \mathrm{mmHg} \\
\mathrm{Cl}>2.5 \mathrm{~L} / \mathrm{min} / \mathrm{m}^{2}\end{array}$ & NA & Vigileo/FT \\
\hline & Control & $A D$ & $A D$ & NA & NA & NA & $\begin{array}{l}\text { Standard } \\
\text { monitoring }\end{array}$ \\
\hline \multirow[t]{2}{*}{ Weinberg [29] } & GDT & $250 \mathrm{~mL}$ & $\begin{array}{l}\text { AD with Crystal- } \\
\text { loids } \\
\text { or Colloids }\end{array}$ & MAP/CI guided & $\begin{array}{l}\mathrm{SWV}<20 \% / 15 \% \\
\mathrm{MAP} \text { of } 20 \% \text { basal } \\
\mathrm{Cl}>2.2 \mathrm{~L} / \mathrm{min} / \mathrm{m}^{2}\end{array}$ & NA & EV1000 \\
\hline & Control & $A D$ & $\begin{array}{l}\text { AD with Crystal- } \\
\text { loids } \\
\text { or Colloids }\end{array}$ & $\begin{array}{l}\text { Discretion of the } \\
\text { anesthetist }\end{array}$ & $\begin{array}{l}\text { CVP }>8 \text { mmHg dur- } \\
\text { ing dissection and } \\
\text { hepatic transection } \\
\text { stages }\end{array}$ & NA & EV1000 \\
\hline
\end{tabular}

$\mathrm{NA}$, data not available; UN, unspecified; AD; at the discretion of the care-giving anesthesiologist; GDT, patients' subgroup receiving goal-directed therapy; mL, milliliters; SV, stroke volume; FTC, corrected flow time; ODP, esophgeal doppler probe; $\mathrm{O}_{2} E R$, oxygen extraction; MAP, mean arterial pressure; UO, urine output; CVP, central venous pressure; SVV, stroke volume variation; FC, fluid challenge; $\mathrm{Cl}$; cardiac index; PAC, pulmonary artery catheter; PAOP; pulmonary artery occlusion pressure

Vigileo/FT; Vigileo/Flow Track: Edwards Lifesciences, Irvine, CA, USA

CardioQ: DP12 probe; Pharmaco NZ, Auckland, New Zealand

ProAQT: PULSION Medical Systems SE, Munich, Germany

LiDCOrapid/ LiDCOplus: LiDCO, Cambridge, UK

value $=0.23$ and $54.8 \%(42.5 ; 67.0)$ vs. $39.8 \%(26.5 ; 53.1)$; $p$ value $=0.09$, respectively $]$.

In the subgroup of those studies reporting an overall rate of complication $<30 \%$, the total amount of perioperative fluids was comparable between patients receiving GDT and controls s [3474 (2313; 4635) vs. 4236 (2765; 5708); $p$ value $=0.40$ ]. The same result was observed for the subgroups of those studies reporting an overall rate of complication between 30 and 50\% [4534 $(2905 ; 6162)$ vs. $4153(3053 ; 5254) ; p$ value $=0.64]$ or above $50 \%$ [ 4723 $(3714 ; 5732)$ vs. $5425(3886 ; 6966) ; p$ value $=0.36]$.

\section{Postoperative outcomes: complications and perioperative mortality}

GDT reduced the rate of postoperative complications (Fig. 2 and Additional file 1: Figure S2) [pooled risk difference GDT vs. controls $(95 \% \mathrm{CI})=-0.10 \quad(-0.14$, $-0.07) ; \mathrm{Chi}_{2}=30.97, p$ value $<0.00001 ; I_{2}(95 \% \mathrm{CI})=19 \%$ $(0-52.1 \%)]$. Considering the rate of organ-related postoperative events, GDT did not reduce neither renal $(p$ value $=0.52)$, nor cardiovascular $(p$ value $=0.86)$ or pulmonary $(p$ value $=0.14)$ or neurological $(p$ value $=0.44)$ or infective $(p$ value $=0.12)$ complications (Additional file 1: Tables S5-S9).

In the 16 studies reporting perioperative mortality, GDT strategy did not show any benefits (Fig. 3 and Additional file 1: Figure S3) [pooled risk difference GDT vs. controls $(95 \% \mathrm{CI})=-0.016(-0.0334 ; 0.0014)$; $\mathrm{Chi}_{2}=3.23, p$ value $\left.=0.07 ; I_{2} 18 \%(0-54.8 \%)\right]$.

\section{Discussion}

The main findings of this systematic review and metaanalysis can be summarized as follows: (1) GDT significantly reduces postoperative complications but not mortality; (2) the effect of GDT on postoperative complications was not modified by the overall amount of fluid infused in the perioperative period.

To the best of our knowledge, this is the most updated and complete systematic review including RCTs comparing patients managed with GDT vs. controls in the perioperative period, as compared to other meta-analyses $[8,9,19]$.

Postoperative complications are common after major surgery and represent an important financial and social burden [5, 6]. The optimization of fluid management has been extensively studied as a potential adjustable 


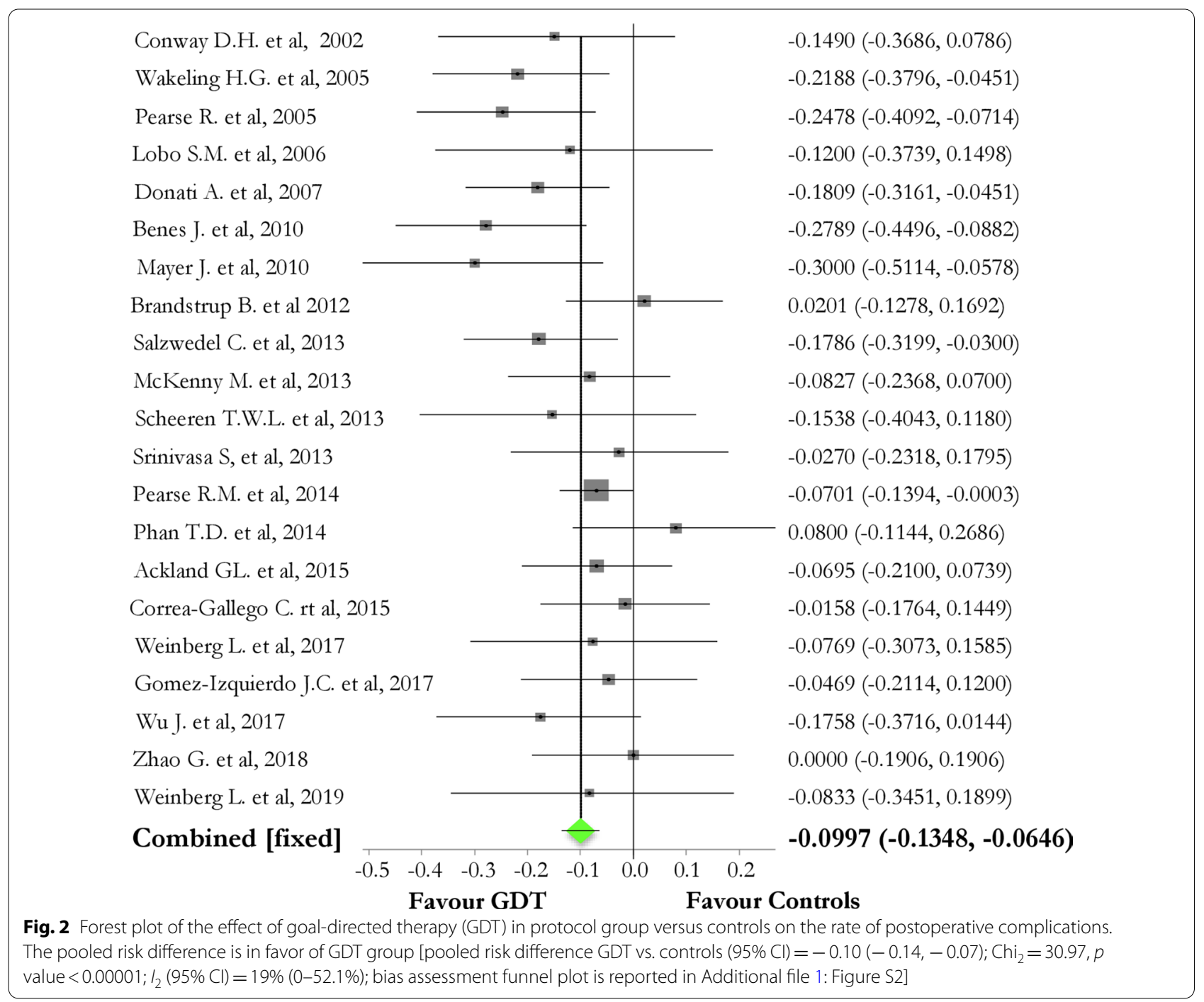

perioperative factor, by adopting specific protocols aimed at optimizing predetermined hemodynamic endpoints [7-13]. This step-by-step GDT process should, in principle, prevent fluid overload (irrespective of the overall amount administered), by closely monitoring the effects on predefined flow or pressure variables of each bolus administered, as long as the individual plateau is achieved $[35,36]$. In other words, during GDT, perioperative overall fluid administration is one of the tools to achieve a predefined endpoint and not the endpoint itself, and its titration should reduce postoperative complications, irrespective of the dose achieved.

In the field of perioperative fluid administration, summarizing the evidence available in the literature into clear clinical suggestions for the daily clinical practice is rather complex. On the one hand, there is a tendency towards a more restrictive approach (as supported by the ERAS pathways [14]). On the other, a recent large RCT performed by Myles et al. challenged this concept, and showed that a median intravenous-fluid intake of $3.7 \mathrm{l}$ as compared to $6.11 \mathrm{did}$ not affect the rate of disability-free survival at one year, being also associated with higher rates of acute kidney injury, surgical-site infection and renalreplacement therapy [15]. The negative results of this trial could be partially explained by the lack of the individualization of fluid administration, which may have contributed to the renal damage, together with the use of vasopressors, low blood pressure and, possibly, dehydration.

Interestingly, GDT trials performed in the period 2000-2010 ago showed important improvements in postoperative morbidity, as compared to more recent ones (Fig. 2). Factors influencing this discrepancy may be related to a different approach to the perioperative fluid balance, along with the advances in all the ERAS components (i.e., avoidance of opioids, early feeding and ambulation and others) and in surgical care (i.e. more 


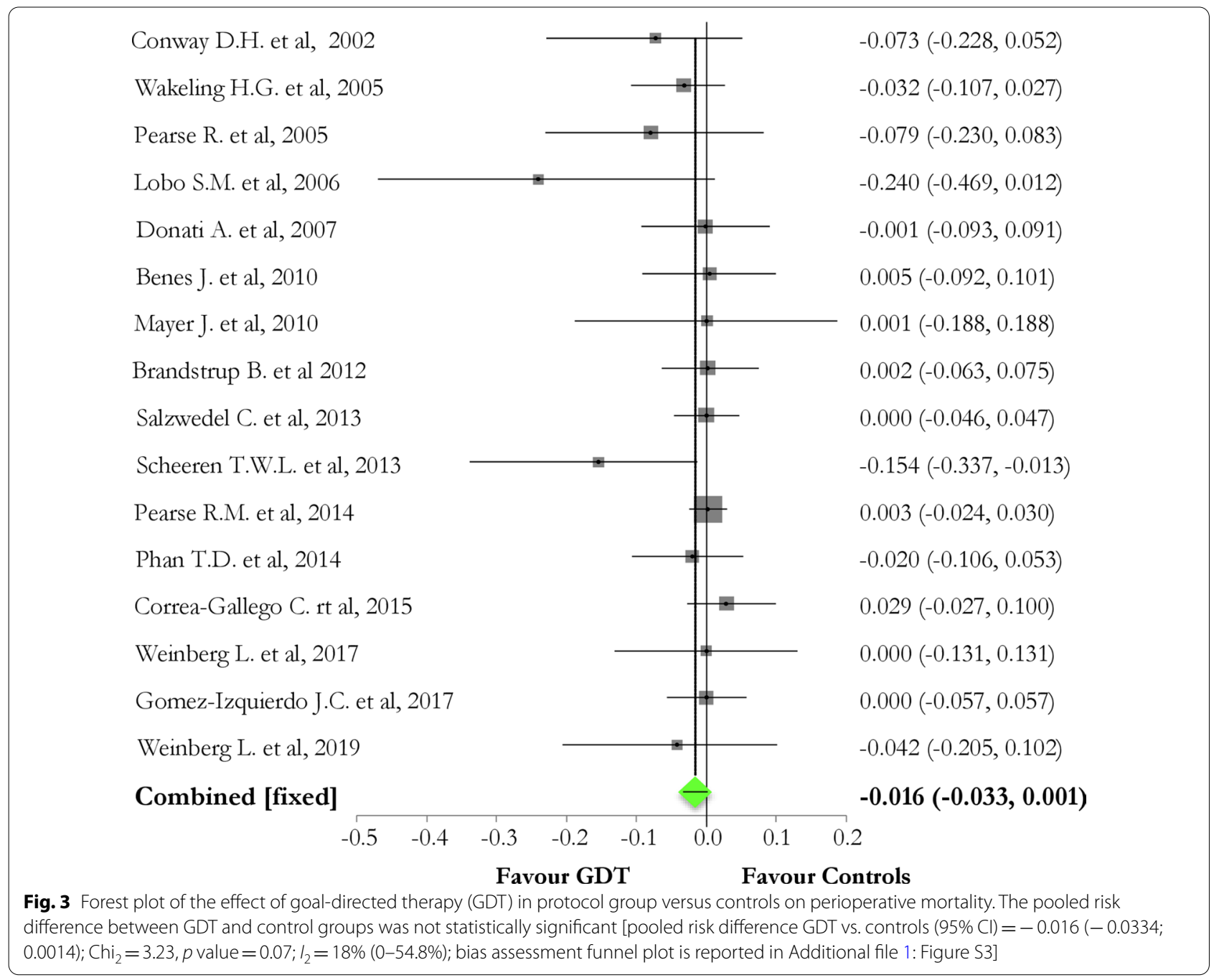

minimally invasive approaches). Our results add to this discussion by suggesting that, in those perioperative scenarios expected to be managed with large amounts of fluids, the use of a GDT approach should be encouraged, since a trend towards the reduction of postoperative complications was shown.

As a matter of fact, by including studies reporting an overall median rate of postoperative complications $>30 \%$, GDT approach reduces overall postoperative complications [pooled risk difference $(95 \% \mathrm{CI})=-0.10(-0.14$, $-0.07) ; p$ value $<0.00001]$, confirming data retrieved from previous findings meta-analyses [8, 9, 19], and encouraging the use of the GDT approach for managing high risk surgical patients.

In this setting, the definition of postoperative complications is crucial, but it is far from being standardized. We only included trials performed in a relatively short timeline, avoiding outdated trials, which may not be representative of current clinical practice.
However, the results of the GDT on overall or organrelated rate of postoperative complications could be related to the quality of data reported in the studies. In fact, both the analyses of the rate of overall postoperative complications and mortality considered the rate of patients who had at least one complication or who died. In contrast, the analysis of organ-related complications considered the number of specific organ-related events. This latter evaluation.

is potentially biased by (1) the definition adopted in the period of enrolment (i.e., the definition of postoperative renal or cardiac dysfunction have been continuously updated [37, 38]); (2) the overlapping of some clinical definitions (i.e., the occurrence of postoperative acute respiratory distress syndrome may be considered as both pulmonary or infective complication); (3) data could be biased by those patients having more than one complication; (4) the gravity of the events potentially biasing the comparability and consistency of pooled data (i.e., 
sepsis or would infection are both postoperative infective events).

On the contrary, we found no difference in perioperative mortality, despite a trend towards its reduction in the GDT subgroup $(p=0.07)$. In a previous systematic review, the use of GDT showed an overall benefit in mortality (OR 0.52, 95\% CI 0.36-0.74; $p=0.003$ ); however, when analyzing the population according to the surgical risk, the authors found that mortality benefit was observed only in the high risk patients subgroup (OR 0.20 , 95\% CI $0.09-0.41 ; p<0.0001)$, whereas it had no effect on intermediate-risk patients (OR $0.83,95 \%$ CI $0.41-1.69 ; p=0.62)$ [8]. This review also included studies performed in a relatively longer timeframe, with some studies reporting high mortality rates. This could be related to the increasing improvement of overall perioperative management strategies, including fluid administration policies $[35,36]$.

\section{Strengths and Limitations of this meta-analysis.}

Our study has some limitations. First, the consistency of data reporting postoperative fluid administration is also somewhat lacking. We considered the amount of fluid administration, including both colloids and crystalloids, since data regarding blood products are not reported in most of the included studies. Moreover, more comprehensive indications regarding the perioperative fluid policy adopted would result from the analysis of fluid balance, which is, unfortunately, reported in only three studies [27, 29,34]. The effect of the type of fluids adopted in the studies may also play a role. Intraoperatively, the GDT subgroup received roughly the same amount of crystalloid and colloids, whereas controls received slightly more crystalloids (56\%). Boluses of colloids have been frequently used to optimize predefined hemodynamic targets, whereas crystalloids have often been infused often as maintenance (Table 3). However,

Table 3 Summary of perioperative fluids administered in the included studies

\begin{tabular}{|c|c|c|c|c|c|c|c|c|}
\hline \multirow[t]{3}{*}{ Study } & \multicolumn{6}{|c|}{ Total intraoperative fluid administration } & \multirow{2}{*}{\multicolumn{2}{|c|}{$\begin{array}{l}\text { Total perioperative } \\
\text { Fluid administration }(95 \% \mathrm{CI})\end{array}$}} \\
\hline & \multicolumn{2}{|c|}{ Colloids (ml) $(95 \% \mathrm{Cl} / \mathrm{SD})$} & \multicolumn{2}{|c|}{ Crystalloids (ml) $(95 \% \mathrm{Cl} / \mathrm{SD})$} & \multicolumn{2}{|c|}{ Intraoperative blood (ml) } & & \\
\hline & GDT & Controls & GDT & Controls & GDT & Controls & GDT & Controls \\
\hline Conway [41] & 1960 (NA) & 1325 (NA) & NA & NA & NA & NA & 4522 (NA) & 3770 (NA) \\
\hline Wakeling [42] & $2000(N A)^{*}$ & $1500(\mathrm{NA})$ & 3000 (NA) & 3000 (NA) & NA & NA & $5000(\mathrm{NA})$ & 4500 (NA) \\
\hline Pearse [30] & $1907(N A)^{*}$ & $930(N A)$ & 1204 (NA) & $960(N A)$ & $125(0-734)$ & $0(0-485)$ & $2837(\mathrm{NA})$ & 2164 \\
\hline Lobo $[26]$ & NA & NA & NA & NA & $713(458)$ & $609(244)$ & $7447(\mathrm{NA})$ & $6877(\mathrm{NA})$ \\
\hline Donati [31] & $1940(673)$ & $1805(611)$ & NA & NA & $260(130)$ & $271(173)$ & $4131(\mathrm{NA})$ & $4014(\mathrm{NA})$ \\
\hline Benes [25] & $1425(1000-1500)$ & $1000(540-1250)$ & $2321(1640-3002)$ & $2459(1529-3389)$ & $0(0-500)$ & $270(0-578)$ & 5333 (NA) & 4987 (NA) \\
\hline Mayer [43] & $1188(638-1738)^{*}$ & $817(350-1284)$ & $2489(1684-3294)$ & $3153(1889-4417)^{*}$ & NA & NA & $\begin{array}{l}4528(2211- \\
6845)\end{array}$ & 4494 (2933-6055) \\
\hline Brandstrup [32] & $810(N A)^{*}$ & 475 (NA) & $483(\mathrm{NA})$ & 443 (NA) & $78(278)$ & $77(407)$ & $6144(N A)$ & 5909 (NA) \\
\hline Salzwedel [27] & $773(109-1437)$ & $724(4-1444)$ & $2862(1646-4078)$ & $2680(1526-3834)$ & $145(371)$ & $224(1036)$ & 7053 (NA) & 7597(NA) \\
\hline McKenny [44] & $\begin{array}{l}1000(1000- \\
1500)^{*}\end{array}$ & $500(0-1000)$ & $1000(787-1750)$ & $2000(1725-2500)^{*}$ & NA & NA & $2620(N A)$ & 2881 (NA) \\
\hline Scheeren [28] & $1589(1150-2028)$ & $927(631-1223)$ & NA & NA & $319(495)$ & $685(832)$ & $\begin{array}{l}4477(3740- \\
5214)\end{array}$ & $4528(3693-5363)$ \\
\hline Srinivasa [45] & $591(471)$ & $297(275)$ & NA & NA & NA & NA & 5744 (NA) & 4014 (NA) \\
\hline Pearse [19] & $1250(1000-2000)$ & $500(0-1000)$ & $1000(459-2000)$ & $2000(1283-3000)$ & NA & NA & $3256(N A)$ & 3100 (NA) \\
\hline Phan [46] & $500(250-750)^{*}$ & $0(0-300)$ & $1500(1000-2000)$ & $1400(1000-2000)$ & NA & NA & $4470(\mathrm{NA})$ & 3916 (NA) \\
\hline Ackland [47] & $500(0-1000)$ & $625(0-1438)$ & $3000(2000-4000)$ & $3000(2000-4000)$ & NA & NA & $5000(\mathrm{NA})$ & $4748(N A)$ \\
\hline Correa-Gallego [48] & NA & NA & NA & NA & NA & NA & 3800 (NA) & $4500(\mathrm{NA})$ \\
\hline Weinberg [34] & $200(500-700)$ & $200(175-550)$ & 1875 (1000-2000) & $4000(2313-4206)^{*}$ & NA & NA & 4516 (NA) & 7034 (NA) \\
\hline $\begin{array}{l}\text { Gomez-Izquierdo } \\
\text { [49] }\end{array}$ & $500(323-687)$ & $\begin{array}{l}2102(1600- \\
2528)^{*}\end{array}$ & $900(400-1400)^{*}$ & $0(0-500)$ & NA & NA & $1819(\mathrm{NA})$ & 2914 (NA) \\
\hline Wu [50] & $900(400-1400)^{*}$ & $0(0-500)$ & $500(323-687)$ & 2102 (1600-2528) & NA & NA & $\begin{array}{l}1535(1000- \\
2272)\end{array}$ & $2370(1779-3071)$ \\
\hline Zhao [33] & $775(539-1011)^{*}$ & $487(435-539)$ & $471(371-571)$ & $459(427-491)$ & $885(377)$ & $443(140)$ & $\begin{array}{l}1478(1167- \\
1790)\end{array}$ & $1183(1120-1246)$ \\
\hline Weinberg yyy[29] & $200(500-700)$ & $200(175-550)$ & $1875(1000-2000)$ & $2000(1125-2000)$ & $\begin{array}{l}356 \\
(248-465)\end{array}$ & $465(465-465)$ & $4950(\mathrm{NA})$ & 4450 (NA) \\
\hline
\end{tabular}

NA, data not available; GDT, patients' subgroup receiving goal-directed therapy; ml, milliliters. Data are reported including $95 \%$ confidence interval (95\%Cl) or standard deviation (SD), as appropriate; when NA is present after a value, it implies a computation of available data reported in the paper. Perioperative fluid therapy reports the overall amount of fluid includes the fluid infused during the operation and within the first $24 \mathrm{~h}$ in the critically ill area. ${ }^{*}=$ difference reported in the study as statistically significant among GDT/controls subgroups regarding intraoperative colloids or crystalloids administration 
a minority of the included studies reported a significant difference in the amount of crystalloids/colloids used between GDT and control subgroups (Table 3). Despite a recent RCT comparing the use of low-molecular-weight hydroxyethylstarch vs. $0.9 \%$ saline in high-risk surgical patients showed no significant difference in postoperative outcomes [39], the debate regarding the optimal perioperative fluid is still ongoing, and the inconsistency of data did not allow any specific subgroup analysis on this topic.

Second, the overall amount of perioperative fluid administration may be biased by the lacking report of oral fluid intake or perioperative maintenance fluid (i.e., sometime reported as $\mathrm{ml} / \mathrm{kg} / \mathrm{hr}$, without providing data regarding the median weight of the population and/or the hours of observation).

Third, the study is limited due to concerns regarding bias in most of the included studies. The different sample sizes of the included studies may add another source of heterogeneity (as confirmed by the interquartile ranges of the $I^{2}$ ). Additionally, the overall quality of the included studies reported "some concerns" in the majority (76.2\%), mostly related to the selection of the reported results due to drawbacks in the trial registration. Moreover, the definition of postoperative complications and the timing in mortality assessment may vary among the included studies, implying a bias in the comparability of the reported outcomes.

Finally, according to previous meta-analyses in this field $[8,40]$, we adopted a database combination search strategy including PUBMED $^{\circledR}$, EMBASE $^{\circledR}$ and the Cochrane Controlled Clinical trials register, excluding different sources (i.e. Web of Science ${ }^{\circledR}$ ). Although this choice should allow a reliable coverage of the published studies for the topic of interest, some RCTs could not be identified.

Despite the abovementioned limitations and the marked heterogeneity in trial quality and design, our results suggest that GDT in the perioperative settings has a significant benefit in reducing rates of complications. This study strengthens the concept that GDT should be routinely applied in perioperative settings, especially in those surgeries in need of high intravascular volume replacement.

\section{Conclusions}

GDT strategy reduces postoperative complications, but not perioperative mortality, irrespectively of perioperative overall amount of fluids infused.

\section{Supplementary Information}

The online version contains supplementary material available at https://doi. org/10.1186/s13054-021-03464-1.

Additional file 1. Table S1: PRISMA-DTA checklist. Table S2: Extracted data in each study assessed for eligibility. Table S3: Full text articles excluded, not fitting eligibility criteria. Table S4: Summary of perioperative cumulative fluid balance in the included studies. Table S5: Renal complications. Table S6: Cardiovascular complications. Table S7: Pulmonary complications. Table S8: Neurological complications. Table S9: Infective complications. Figure S1: Risk of bias assessment of the included studies. Figure S2: Bias assessment plot. Overall complications. Figure S3: Bias assessment plot. Overall mortality.

\section{Abbreviations}

GDT: goal-directed therapy; RCT: randomized-controlled trial; ERAS: enhanced recovery after surgery; SD: standard deviation; 95\% Cl: 95\% confidence intervals.

\section{Acknowledgements}

We are thankful to Dr. Katerina Negri for the linguistic revision of this manuscript.

\section{Authors' contributions}

A.M. and C.R. designed the study, conducted the primary data search, solved the classification discrepancies in data collection and drafted the manuscript; L.C., D.Z., F.I., E.M., S.S., D.B. and M.B. performed the evaluation of titles and abstracts and helped in data collection and the manuscript preparation, L.S. and G.S. conducted the data analysis and helped in manuscript preparation; P.P. substantially contributed in manuscript preparation and data interpretation; M.C. helped in designing the study and in the primary search and substantially contributed in manuscript preparation and data interpretation. All the authors approved the final version of the paper and agree to be accountable for all aspects of the work, thereby ensuring that questions related to the accuracy or integrity of any part of the work are appropriately investigated and resolved. All authors read and approved the final manuscript.

Funding

This work has not been funded by an external source.

\section{Availability of data and materials}

The datasets used and/or analyzed during the current study are available from the corresponding author on reasonable request.

Ethical approval and consent to participate

Not applicable.

\section{Consent for publication}

Not applicable.

\section{Competing interests}

Dr. Messina received travel expenses and registration for meetings, congresses, and courses and lecture fees from Vygon; Prof. Cecconi is a consultant for Edwards Lifesciences, LiDCO and Cheetah Medical.

\footnotetext{
Author details

${ }^{1}$ Humanitas Clinical and Research Center - IRCCS, Rozzano, MI, Italy. ${ }^{2}$ Department of Biomedical Sciences, Humanitas University, Pieve Emanuele, MI, Italy. ${ }^{3}$ Anesthesia and Intensive Care, San Martino Policlinico Hospital, IRCCS for Oncology and Neuroscience, Genoa, Italy. ${ }^{4}$ Department of Surgical Sciences and Integrated Diagnostic (DISC), University of Genoa, Genoa, Italy. ${ }^{5}$ Anesthesia and Intensive Care Medicine, Maggiore Della Carità University Hospital, Novara, Italy. ${ }^{6}$ Anesthesia and Intensive Care Medicine, Azienda Sanitaria Universitaria Integrata Udine, Udine, Italy. ${ }^{7}$ Clinical Epidemiology
} 
and Medical Statistics Unit, Department of Medical, Surgical and Experimental, University of Sassari, Sassari, Italy.

Received: 28 October 2020 Accepted: 7 January 2021 Published online: 01 February 2021

\section{References}

1. Jhanji S, Thomas B, Ely A, Watson D, Hinds CJ, Pearse RM. Mortality and utilisation of critical care resources amongst high-risk surgical patients in a large nhs trust. Anaesthesia. 2008;63:695-700.

2. Thompson JS, Baxter BT, Allison JG, Johnson FE, Lee KK, Park WY. Temporal patterns of postoperative complications. Arch Surg. 2003;138:596-602; discussion 602-593.

3. Tevis SE, Kennedy GD. Postoperative complications and implications on patient-centered outcomes. J Surg Res. 2013;181:106-13.

4. Pearse RM, Moreno RP, Bauer P, Pelosi P, Metnitz P, Spies C, et al. Mortality after surgery in europe: A 7 day cohort study. Lancet. 2012;380:1059-65.

5. Khuri SF, Henderson WG, DePalma RG, Mosca C, Healey NA, Kumbhani $D J$, et al. Determinants of long-term survival after major surgery and the adverse effect of postoperative complications. Ann Surg. 2005;242:32641; discussion 341-323.

6. Healy MA, Mullard AJ, Campbell DA Jr, Dimick JB. Hospital and payer costs associated with surgical complications. JAMA Surg. 2016;151:823-30.

7. Holte K, Kehlet H. Fluid therapy and surgical outcomes in elective surgery: a need for reassessment in fast-track surgery. J Am Coll Surg. 2006;202:971-89.

8. Cecconi M, Corredor C, Arulkumaran N, Abuella G, Ball J, Grounds RM, et al. Clinical review: Goal-directed therapy-what is the evidence in surgical patients? The effect on different risk groups. Crit Care. 2013;17:209.

9. Hamilton MA, Cecconi M, Rhodes A. A systematic review and metaanalysis on the use of preemptive hemodynamic intervention to improve postoperative outcomes in moderate and high-risk surgical patients. Anesth Analg. 2011;112:1392-402.

10. Lobo SM, de Oliveira NE. Clinical review: What are the best hemodynamic targets for noncardiac surgical patients? Crit Care. 2013;17:210.

11. Marik PE. Perioperative hemodynamic optimization: a revised approach. J Clin Anesth. 2014;26:500-5.

12. Voldby AW, Brandstrup B. Fluid therapy in the perioperative setting-a clinical review. J Intensive Care. 2016;4:27.

13. Thacker JK, Mountford WK, Ernst FR, Krukas MR, Mythen MM. Perioperative fluid utilization variability and association with outcomes: considerations for enhanced recovery efforts in sample us surgical populations. Ann Surg. 2016;263:502-10.

14. Gustafsson UO, Scott MJ, SchwenkW, Demartines N, Roulin D, Francis $\mathrm{N}$, et al. Guidelines for perioperative care in elective colonic surgery: enhanced recovery after surgery (eras((r))) society recommendations. World J Surg. 2013;37:259-84

15. Myles PS, Bellomo R, Corcoran T, Forbes A, Peyton P, Story D, et al. Restrictive versus liberal fluid therapy for major abdominal surgery. N Engl J Med. 2018;378:2263-74.

16. Miller TE, Myles PS. Perioperative fluid therapy for major surgery. Anesthesiology. 2019;130:825-32.

17. Wrzosek A, Jakowicka-Wordliczek J, Zajaczkowska R, Serednicki WT, Jankowski M, Bala MM, et al. Perioperative restrictive versus goal-directed fluid therapy for adults undergoing major non-cardiac surgery. Cochrane Database Syst Rev. 2019;12:CD012767.

18. Kaufmann T, Saugel B, Scheeren TWL. Perioperative goal-directed therapy - what is the evidence? Best Pract Res Clin Anaesthesiol. 2019;33:179-87.

19. Pearse RM, Harrison DA, MacDonald N, Gillies MA, Blunt M, Ackland G, et al. Effect of a perioperative, cardiac output-guided hemodynamic therapy algorithm on outcomes following major gastrointestinal surgery: a randomized clinical trial and systematic review. JAMA. 2014;311:2181-90.

20. Calvo-Vecino JM, Ripolles-Melchor J, Mythen MG, Casans-Frances R, Balik A, Artacho JP, et al. Effect of goal-directed haemodynamic therapy on postoperative complications in low-moderate risk surgical patients: a multicentre randomised controlled trial (fedora trial). Br J Anaesth. 2018;120:734-44.
21. Rollins KE, Lobo DN. Intraoperative goal-directed fluid therapy in elective major abdominal surgery: a meta-analysis of randomized controlled trials. Ann Surg. 2016;263:465-76.

22. Moher D, Liberati A, Tetzlaff J, Altman DG, Group P. Preferred reporting items for systematic reviews and meta-analyses: the prisma statement. BMJ. 2009;339:b2535.

23. Sterne JAC, Savović J, Page MJ, Elbers RG, Blencowe NS, Boutron I, et al. Rob 2: A revised tool for assessing risk of bias in randomised trials. BMJ. 2019:366:14898.

24. Higgins JP, Thompson SG, Deeks JJ, Altman DG. Measuring inconsistency in meta-analyses. BMJ. 2003;327:557-60.

25. Benes J, Chytra I, Altmann P, Hluchy M, Kasal E, Svitak R, et al. Intraoperative fluid optimization using stroke volume variation in high risk surgical patients: Results of prospective randomized study. Crit Care. 2010;14:R118.

26. Lobo SM, Lobo FR, Polachini CA, Patini DS, Yamamoto AE, de Oliveira $\mathrm{NE}$, et al. Prospective, randomized trial comparing fluids and dobutamine optimization of oxygen delivery in high-risk surgical patients [isrctn42445141]. Crit Care. 2006;10:R72.

27. Salzwedel C, Puig J, Carstens A, Bein B, Molnar Z, Kiss K, et al. Perioperative goal-directed hemodynamic therapy based on radial arterial pulse pressure variation and continuous cardiac index trending reduces postoperative complications after major abdominal surgery: A multi-center, prospective, randomized study. Crit Care. 2013;17:R191.

28. Scheeren TW, Wiesenack C, Gerlach H, Marx G. Goal-directed intraoperative fluid therapy guided by stroke volume and its variation in high-risk surgical patients: A prospective randomized multicentre study. J Clin Monit Comput. 2013;27:225-33.

29. Weinberg L, lanno D, Churilov L, McGuigan S, Mackley L, Banting J, et al. Goal directed fluid therapy for major liver resection: a multicentre randomized controlled trial. Ann Med Surg (Lond). 2019;45:45-53.

30. Pearse R, Dawson D, Fawcett J, Rhodes A, Grounds RM, Bennett ED. Early goal-directed therapy after major surgery reduces complications and duration of hospital stay. A randomised, controlled trial [isrctn38797445]. Crit Care. 2005;9:R687-693.

31. Donati A, Loggi S, Preiser JC, Orsetti G, Munch C, Gabbanelli V, et al. Goaldirected intraoperative therapy reduces morbidity and length of hospital stay in high-risk surgical patients. Chest. 2007;132:1817-24.

32. Brandstrup B, Svendsen PE, Rasmussen M, Belhage B, Rodt SA, Hansen B, et al. Which goal for fluid therapy during colorectal surgery is followed by the best outcome: Near-maximal stroke volume or zero fluid balance? $\mathrm{Br}$ Anaesth. 2012;109:191-9.

33. Guoliang Zhao* PP, Yinyan Z, Junjie L, Haiyan J, Jianlin S. The accuracy and effectiveness of goal directed fluid therapy in plateau-elderly gastrointestinal cancer patients: a prospective randomized controlled trial. Int J Clin Exp Med. 2018;11:8516-8522.

34. Weinberg L, lanno D, Churilov L, Chao I, Scurrah N, Rachbuch C, et al. Restrictive intraoperative fluid optimisation algorithm improves outcomes in patients undergoing pancreaticoduodenectomy: a prospective multicentre randomized controlled trial. PLoS ONE. 2017;12:e0183313.

35. Heming N, Moine P, Coscas R, Annane D. Perioperative fluid management for major elective surgery. Br J Surg. 2020;107:e56-62.

36. Kampmeier TG, Ertmer C. Individualized goal-directed therapy: the challenge with the fluids. Anesth Analg. 2020;130:596-8.

37. Kellum JA, Lameire N. Group KAKIGW: Diagnosis, evaluation, and management of acute kidney injury: a kdigo summary (part 1). Crit Care. 2013;17:204

38. Sellers D, Srinivas C, Djaiani G. Cardiovascular complications after noncardiac surgery. Anaesthesia. 2018;73(Suppl 1):34-42.

39. Futier E, Garot M, Godet T, Biais M, Verzilli D, Ouattara A, et al. Effect of hydroxyethyl starch vs saline for volume replacement therapy on death or postoperative complications among high-risk patients undergoing major abdominal surgery: the flash randomized clinical trial. JAMA. 2020;323:225-36.

40. Bednarczyk JM, Fridfinnson JA, Kumar A, Blanchard L, Rabbani R, Bell D, et al. Incorporating dynamic assessment of fluid responsiveness into goal-directed therapy: a systematic review and meta-analysis. Crit Care Med. 2017:45:1538-45.

41. Conway DH, Mayall R, Abdul-Latif MS, Gilligan S, Tackaberry C. Randomised controlled trial investigating the influence of intravenous fluid 
titration using oesophageal doppler monitoring during bowel surgery. Anaesthesia. 2002;57:845-9.

42. Wakeling HG, McFall MR, Jenkins CS, Woods WG, Miles WF, Barclay GR, et al. Intraoperative oesophageal doppler guided fluid management shortens postoperative hospital stay after major bowel surgery. Br J Anaesth. 2005;95:634-42.

43. Mayer J, Boldt J, Mengistu AM, Rohm KD, Suttner S. Goal-directed intraoperative therapy based on autocalibrated arterial pressure waveform analysis reduces hospital stay in high-risk surgical patients: a randomized, controlled trial. Crit Care. 2010;14:R18.

44. McKenny M, Conroy P, Wong A, Farren M, Gleeson N, Walsh C, et al. A randomised prospective trial of intra-operative oesophageal dopplerguided fluid administration in major gynaecological surgery. Anaesthesia. 2013;68:1224-31.

45. Srinivasa S, Taylor MH, Singh PP, Yu TC, Soop M, Hill AG. Randomized clinical trial of goal-directed fluid therapy within an enhanced recovery protocol for elective colectomy. Br J Surg. 2013;100:66-74.

46. Phan TD, D'Souza B, Rattray MJ, Johnston MJ, Cowie BS. A randomised controlled trial of fluid restriction compared to oesophageal dopplerguided goal-directed fluid therapy in elective major colorectal surgery within an enhanced recovery after surgery program. Anaesth Intensive Care. 2014:42:752-60.
47. Ackland GL, Iqbal S, Paredes LG, Toner A, Lyness C, Jenkins N, et al. Individualised oxygen delivery targeted haemodynamic therapy in high-risk surgical patients: a multicentre, randomised, double-blind, controlled, mechanistic trial. Lancet Respir Med. 2015;3:33-41.

48. Correa-Gallego C, Tan KS, Arslan-Carlon V, Gonen M, Denis SC, LangdonEmbry L, et al. Goal-directed fluid therapy using stroke volume variation for resuscitation after low central venous pressure-assisted liver resection: a randomized clinical trial. J Am Coll Surg. 2015;221:591-601.

49. Gómez-Izquierdo JC, Trainito A, Mirzakandov D, Stein BL, Liberman S, Charlebois $\mathrm{P}$, et al. Goal-directed fluid therapy does not reduce primary postoperative ileus after elective laparoscopic colorectal surgery: a randomized controlled trial. Anesthesiology. 2017;127:36-49.

50. Jie Wu YM, Tianlong W, Geng X, Long F, Ying Z. Goal-directed fluid management based on the auto-calibrated arterial pressure-derived stroke volume variation in patients undergoing supratentorial neoplasms surgery. Int J Clin Exp Med. 2017;10:3106-14.

\section{Publisher's Note}

Springer Nature remains neutral with regard to jurisdictional claims in published maps and institutional affiliations.
Ready to submit your research? Choose BMC and benefit from:

- fast, convenient online submission

- thorough peer review by experienced researchers in your field

- rapid publication on acceptance

- support for research data, including large and complex data types

- gold Open Access which fosters wider collaboration and increased citations

- maximum visibility for your research: over $100 \mathrm{M}$ website views per year

At BMC, research is always in progress.

Learn more biomedcentral.com/submissions 